\title{
MODIFICATIONS TO THE CAVOPULMONARY ANASTOMOSIS DO NOT ELIMINATE EARLY SINUS NODE DYSFUNCTION
}

Mitchell I. Cohen, MD

Nancy D. Bridges, $\mathrm{MD}^{\mathrm{a}}$

J. William Gaynor, $\mathrm{MD}^{\mathrm{b}}$

Timothy M. Hoffman, MD

Gil Wernovsky, MD

Victoria L. Vetter, $\mathrm{MD}^{\mathrm{a}}$

Thomas L. Spray, MD ${ }^{\mathrm{b}}$

Larry A. Rhodes, MD
Objective: To determine whether operations that theoretically jeopardize the sinus node (hemi-Fontan and/or lateral tunnel Fontan procedures) are associated with a greater risk of sinus node dysfunction than those that theoretically spare the sinus node (bidirectional Glenn and/or extracardiac conduit).

Methods: Between January 1, 1996, and December 31, 1999, a prospective cohort study was conducted evaluating the incidence of sinus node dysfunction in patients undergoing a bidirectional Glenn or hemi-Fontan procedure and those in whom the Fontan repair was completed with either an extracardiac conduit or a lateral tunnel. Sinus node dysfunction was defined (1) as a heart rate more than 2 SD below age-adjusted norms or (2) as a predominant junctional rhythm and/or a sinus pause of more than 3 seconds as determined by the resting electrocardiogram and/or ambulatory monitoring at hospital discharge.

Results: Fifty-one patients had a bidirectional Glenn shunt (mean age $7.8 \pm$ 5.1 months) and 79 a hemi-Fontan procedure (mean age $6.9 \pm 2.8$ months). The incidence of sinus node dysfunction on postoperative day 1 was significantly higher after the hemi-Fontan $(36 \%)$ than after the bidirectional Glenn shunt $(9.8 \%)$; however, by hospital discharge this difference was no longer apparent (hemi-Fontan [8\%]; bidirectional Glenn [6\%]; $P=$ not significant). No difference in early sinus node dysfunction was discernible after the extracardiac conduit (4/30 [13\%]) compared with the lateral tunnel Fontan procedure $(6 / 46[13 \%])(P=$ not significant). No diagnostic or perioperative variables were predictive of sinus node dysfunction.

Conclusions: Avoidance of surgery near the sinus node has no discernible effect on the development of early sinus node dysfunction. Thus, concerns about early sinus node dysfunction should not override patient anatomy or surgeon preference as determinants of which cavopulmonary anastomosis to perform. (J Thorac Cardiovasc Surg 2000;120:891-901)
From the Divisions of Cardiology ${ }^{\mathrm{a}}$ and Cardiothoracic Surgery ${ }^{\mathrm{b}}$ and The Departments of Pediatrics and Surgery, The Children's Hospital of Philadelphia and The University of Pennsylvania School of Medicine, Philadelphia, Pa.

Read at the Eightieth Annual Meeting of The American Association for Thoracic Surgery, Toronto, Ontario, Canada, April 30-May 3, 2000

Received for publication May 4, 2000; revisions requested June 13, 2000; revisions received June 20, 2000; accepted for publication June 26, 2000.

Address for reprints: Mitchell I. Cohen, MD, Division of Pediatric Cardiology, The Children's Hospital of Philadelphia, 34th \& Civic Center Blvd, Philadelphia, PA 19104 (E-mail: cohenmi@email.chop.edu)

Copyright (C) 2000 by The American Association for Thoracic Surgery

0022-5223/2000 $\$ 12.00+0 \quad \mathbf{1 2 / 6 / 1 0 9 7 0 8}$

doi:10.1067/mtc. 2000.109708
The anastomosis between the superior vena cava (SVC) and the pulmonary artery is a frequently used intermediate step in the surgical staging of patients with congenital heart defects unsuitable for a biventricular repair. ${ }^{1-4}$ The addition of an intermediate cavopulmonary connection has been shown to reduce the volume load on the single ventricle and decrease overall mortality. ${ }^{4-7}$ There are two choices of second-stage procedures, the hemi-Fontan operation and the bidirectional Glenn shunt. Although the hemi-Fontan procedure facilitates completion of the lateral tunnel, there is concern that the sinus node artery, which courses in the superior cavoatrial junction, is susceptible to injury with this procedure. Sinus node dysfunction has been 
reported in $20 \%$ to $25 \%$ of patients early after the Fontan operation and is a predictor of late sinus node dysfunction. ${ }^{8,9}$

Sinus node dysfunction may decrease cardiac output either by limiting heart rate in patients with a relatively fixed stroke volume or by decreasing diastolic ventricular filling as a result of junctional rhythm and loss of atrioventricular synchrony. Some investigators have speculated that alternative approaches such as the bidirectional Glenn and extracardiac conduit Fontan, which minimize cavoatrial incisions, may reduce the incidence of sinus node dysfunction. ${ }^{9-11}$ Because sinus node dysfunction increases with time from the Fontan operation, ${ }^{9}$ previous studies evaluating surgical modifications have been limited by comparing procedures from different time periods, with varying lengths of follow-up, and different noninvasive techniques for assessing sinus node function. ${ }^{8}$ There are currently no studies that have prospectively compared modifications to the cavopulmonary anastomosis with respect to sinus node dysfunction.

The objective of this study was to determine whether operations that theoretically jeopardize the sinus node or its blood supply (specifically, the hemi-Fontan and/or lateral tunnel Fontan procedures) are associated with a greater risk of sinus node dysfunction than those that theoretically spare the blood supply to the sinus node artery (specifically, the bidirectional Glenn shunt and/or extracardiac conduit Fontan operations).

\section{Methods}

Patients. A prospective cohort study was performed between January 1, 1996, and December 31, 1999, evaluating the early incidence of sinus node dysfunction in all patients undergoing an initial superior cavopulmonary anastomosis with or without a subsequent modified Fontan procedure. All procedures were performed at The Children's Hospital of Philadelphia. Our general approach has been to perform a systematically staged fenestrated Fontan operation in all patients with single ventricle physiology regardless of preoperative risk factors. The decision of which cavopulmonary anastomosis and subsequent Fontan operation to perform was at the surgeon's discretion without randomization. Patients who underwent an initial hemi-Fontan or bidirectional Glenn shunt at an outside institution $(n=3)$, a patient with sinus node dysfunction before the superior cavopulmonary anastomosis $(\mathrm{n}=1)$, and patients who underwent a nonstaged Fontan procedure during this period $(\mathrm{n}=9)$ were excluded from the study.

To determine the early incidence of sinus node dysfunction, we obtained a resting electrocardiogram (ECG) before each surgical stage, on the first postoperative day, and again at hospital discharge. Ambulatory 24-hour monitoring was performed within 7 days before each of the surgical stages and then again at hospital discharge. If a Holter monitor was not available, the resting ECG served as the principal assessment of sinus node function. To determine possible predictors of sinus node dysfunction, we performed a univariate analysis on a variety of patient, procedural, and time-related variables (Tables I to III).

Operative techniques. During the hemi-Fontan procedure, an incision is made in the superior-anterior aspect of the right atrium and carried medially across the cavoatrial junction onto the SVC. Another longitudinal incision is made in the anterior aspect of the pulmonary arteries to open the pulmonary bifurcation widely. The right pulmonary artery is then anastomosed in a side-to-side fashion to the posterior lip of the opened SVC. A pulmonary artery homograft patch is tailored to create a "dam" separating the pulmonary arteries and venae cavae from the right atrium. ${ }^{12}$

The lateral tunnel Fontan operation consists of a longitudinal right atriotomy incision and excision of the homograft "dam" between the SVC and the right atrium. A 10-mm polytetrafluoroethylene (PTFE) graft" is cut and used as a baffle to create a lateral tunnel connecting the inferior vena cava (IVC) along the right lateral wall of the atrium to the SVC entrance into the pulmonary artery. The anterior suture line is used to close the right atriotomy incision.

An alternative superior cavopulmonary connection involves the creation of a bidirectional Glenn shunt. In the bidirectional Glenn shunt, an incision is made in the anterior aspect of the pulmonary artery and extended onto the right main pulmonary artery and onto the left pulmonary artery posterior to the aorta. The SVC is transected about $1 \mathrm{~cm}$ above the junction of the right atrium, and the cardiac end is oversewn. The cephalic end of the SVC is opened anteriorly and the posterior aspect is sutured to the right pulmonary artery. A homograft patch is tailored to the appropriate size and used to augment the anastomosis from the right pulmonary artery to the pulmonary artery confluence when branch pulmonary artery stenosis is present.

The extracardiac conduit involves separating the IVC from the right atrium and anastomosing an appropriately sized PTFE graft in an end-to-end fashion with the IVC. The conduit is then anastomosed to the underside of the pulmonary arteries, and a homograft patch is used to augment the anastomosis as necessary.

A fenestration was generally created by one of three techniques: (1) single 4-mm punch in the lateral tunnel, (2) single $4.8-\mathrm{mm}$ side-to-side fenestration between the right atrium and the extracardiac conduit, or (3) use of a small tube graft between the systemic venous pathway and pulmonary venous atria.

Definitions. Sinus node dysfunction was defined according to previously established criteria ${ }^{9,13,14}$ : (1) minimum or mean heart rate more than 2 SD below the age-adjusted mean, (2) predominant junctional rhythm, and/or (3) sinus pause of 3

*Gore-Tex graft; registered trade name of W. L. Gore \& Associates, Inc, Flagstaff, Ariz. 


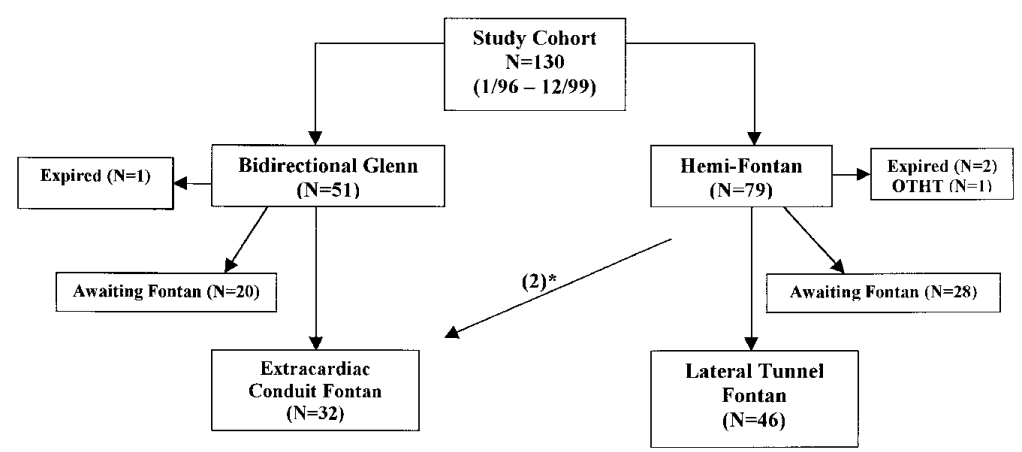

Fig 1. Surgical approach to superior cavopulmonary anastomosis (bidirectional Glenn and hemi-Fontan) and Fontan (extracardiac conduit and lateral tunnel) procedures between 1996 and 1999. The 2 patients $\left(^{*}\right)$ who crossed over were analyzed separately from the Fontan completion groups. OTHT, Orthotopic heart transplantation.

seconds or more in duration. In patients with complete heart block, sinus node dysfunction was based on an underlying atrial rate of more than 2 SD below the age-adjusted mean. The P-wave axis on the ECG obtained before the superior cavopulmonary anastomosis was defined as the patient's intrinsic atrial rhythm. An ectopic atrial rhythm was defined as a change in the $\mathrm{P}$-wave axis quadrant by $30^{\circ}$ or more compared with the baseline ECG before the cavopulmonary operation. Patients with an ectopic atrial rhythm were categorized separately from those with sinus node dysfunction. All ECGs and ambulatory monitors were independently reviewed by two observers (L.A.R. and M.I.C.).

Statistical analysis. Values are reported as mean \pm 2 SD or median with ranges shown. The primary outcome variable was the presence or absence of sinus node dysfunction. On the basis of a projected incidence of sinus node dysfunction of $20 \%$ (according to a review of the literature), ${ }^{8,9}$ we calculated a sample size requirement of at least 38 patients in each group to demonstrate a relative risk of 2 or more, with an alpha of 0.05 and a power of $80 \%$. The "exposed" group was defined as patients undergoing a hemi-Fontan operation, a lateral tunnel Fontan operation, or both operations. The "unexposed" or control group was defined as those patients undergoing a bidirectional Glenn shunt, with or without a subsequent extracardiac conduit Fontan operation. Relative risk and confidence intervals are shown. Comparison of continuous variables between patients with and without sinus node dysfunction was performed by means of the 2-sample $t$ test with equal variances. Categoric variables were analyzed by use of $\chi^{2}$ analysis or the Fisher exact test.

\section{Results}

Study population. A total of 130 patients ( 87 boys, 43 girls) met inclusion criteria and were followed up prospectively (Fig 1). A total of 51 patients underwent a bidirectional Glenn shunt, 30 of whom successfully underwent an extracardiac conduit Fontan operation. Among 79 patients who underwent a hemi-Fontan pro- cedure, 46 went on to have a lateral tunnel Fontan operation. Mortality after the superior cavopulmonary anastomosis was $2.3 \%$ ( 3 patients). Two patients with an initial hemi-Fontan operation underwent a subsequent extracardiac conduit Fontan. These 2 patients were excluded from the primary analysis. The remaining 48 patients had not undergone a Fontan procedure by the end of the observation period.

Cardiac anatomy was classified into 6 groups defined by previous investigators (Table I). ${ }^{15}$ Hypoplastic left heart syndrome was present in $37 \%$ of the study cohort and was not disproportionately represented in any of the surgical groups. Heterotaxy syndrome comprised $12 \%$ of the study group and was more prevalent in the patients undergoing a bidirectional Glenn shunt $(P<$ $.05)$. Of the 51 patients having a bidirectional Glenn shunt, 10 had bilateral SVCs that required a bilateral bidirectional Glenn procedure. Five patients in the hemi-Fontan group who had bilateral SVCs underwent a bidirectional Glenn shunt on the contralateral side of the hemi-Fontan procedure. One patient in each of the bidirectional Glenn and hemi-Fontan groups had an interrupted IVC with azygos continuation. No differences were detected between the study groups with regard to the number of palliative procedures performed before the cavopulmonary anastomosis or age at the time of either surgical stage.

The extracardiac conduit was fenestrated in 29 (97\%) patients. The lateral tunnel Fontan was fenestrated in 43 patients $(93 \%)$ with a single $4-\mathrm{mm}$ punch in the PTFE baffle. The perioperative and operative variables of the two study groups are shown in Tables II and III. There were no significant preoperative or perioperative hemodynamic differences between the patients with a bidirectional Glenn or hemi-Fontan procedure and those in whom the Fontan repair was completed with 
Table I. Demographic data on study cohort $(N=130)$

\begin{tabular}{|c|c|c|c|c|}
\hline Patient related demographic data & $\begin{array}{c}B D G \\
(n=51)\end{array}$ & $\begin{array}{c}H F \\
(n=79)\end{array}$ & $\begin{array}{c}B D G / E C \\
(n=30)\end{array}$ & $\begin{array}{l}H F / L T \\
(n=46)\end{array}$ \\
\hline Male/female & $34 / 17$ & $53 / 26$ & $22 / 8$ & $30 / 16$ \\
\hline \multicolumn{5}{|l|}{ Anatomic subtype of single ventricle } \\
\hline Single, LV, NRGA & $7(14 \%)$ & $6(8 \%)$ & $5(17 \%)$ & $4(9 \%)$ \\
\hline Single LV, TGA & $5(10 \%)$ & $9(11 \%)$ & $2(6 \%)$ & $6(13 \%)$ \\
\hline Single RV (not HLHS) & $13(25 \%)$ & $12(16 \%)$ & $7(24 \%)$ & $9(20 \%)$ \\
\hline Heterotaxy & $11(21 \%)$ & $5(6 \%)$ & $5(17 \%)$ & $2(4 \%)$ \\
\hline HLHS & $10(20 \%)$ & $38(48 \%)$ & $10(33 \%)$ & $19(41 \%)$ \\
\hline Other & $5(10 \%)$ & $9(11 \%)$ & $1(3 \%)$ & $6(13 \%)$ \\
\hline \multicolumn{5}{|l|}{ Palliative procedures prior to $\mathrm{CPA}$} \\
\hline Stage I palliation* & 20 & 63 & 14 & 36 \\
\hline Septectomy $\dagger$ & 28 & 67 & 17 & 37 \\
\hline PA band & 7 & 3 & 1 & 1 \\
\hline Shunt alone & 16 & 13 & 9 & 9 \\
\hline PA plasty \& shunt & 2 & 1 & 1 & 1 \\
\hline Balloon aortic valvotomy & 0 & 1 & 0 & 1 \\
\hline Tricuspid valvuloplasty & 0 & 1 & 0 & 1 \\
\hline None & 7 & 4 & 2 & 9 \\
\hline \multicolumn{5}{|c|}{ Additional procedures at the time of the CPA } \\
\hline Atrial septectomy & 3 & 6 & 3 & 4 \\
\hline Damus-Kaye-Stansel & 3 & 0 & 2 & 0 \\
\hline MPA oversewn & 6 & 4 & 2 & 4 \\
\hline Tricuspid valvuloplasty & 0 & 2 & 0 & 1 \\
\hline Neoaortic Reconstruction & 1 & 1 & 1 & 1 \\
\hline Pacemaker implantation & 0 & 1 & 0 & 0 \\
\hline \multicolumn{5}{|c|}{ Additional procedures at the time of the Fontan } \\
\hline MPA oversewn & & & 2 & 1 \\
\hline Tricuspid valvuloplasty & & & 1 & 4 \\
\hline Atrial septectomy & & & 1 & 5 \\
\hline Age at CPA (mo) & $7.8 \pm 5.1$ & $6.9 \pm 2.8$ & $6.8 \pm 2.6$ & $7.3 \pm 2.6$ \\
\hline Age at Fontan $(\mathrm{mo})$ & & & $23.0 \pm 5.8$ & $23.9 \pm 2.6$ \\
\hline
\end{tabular}

either an extracardiac conduit or lateral tunnel Fontan procedure. Cardiopulmonary bypass times were significantly $(P<.01)$ longer in patients having a hemiFontan compared with a bidirectional Glenn operation and in those having an extracardiac conduit compared with a lateral tunnel Fontan operation. No significant difference was found in the frequency or duration of deep hypothermic circulatory arrest between any of the surgical groups. Twenty-three patients had additional procedures performed at the time of the intermediate cavopulmonary anastomosis and 11 patients at the time of the Fontan operation (Table I). The duration of chest tube drainage and hospitalization did not differ between the two groups either at the intermediate cavopulmonary anastomosis or at the Fontan operation. The median length of stay after the Fontan operation was 5 days.
Perioperative rhythm. All patients had a resting ECG before the operation and on the first postoperative day after each of the surgical stages. Ambulatory 24hour monitors were available in $97(75 \%)$ patients (41/51 bidirectional Glenn; 56/79 hemi-Fontan; $P=$ not significant $[\mathrm{NS}]$ ) before the superior cavopulmonary anastomosis and on 117 (90\%) patients (47 bidirectional Glenn; 70 hemi-Fontan; $P=$ NS) at hospital discharge. Ambulatory 24-hour monitors were available after the Fontan operation in $71(93 \%)$ patients (27 bidirectional Glenn/extracardiac conduit; 44 hemiFontan/lateral tunnel; $P=$ NS). No differences were detected between the groups with respect to the number of Holter monitors used as an assessment of sinus node dysfunction.

The incidence of sinus node dysfunction on postoperative day 1 was significantly higher after the hemi- 
Table II. Superior cavopulmonary connection: Preoperative and perioperative hemodynamics

\begin{tabular}{|c|c|c|}
\hline & $B D G(n=51)$ & $H F(n=79)$ \\
\hline \multicolumn{3}{|l|}{ Perioperative } \\
\hline \multicolumn{3}{|l|}{ Pre-CPA cardiac catheterization } \\
\hline SVC saturation $(\%)$ & $53 \pm 9$ & $49 \pm 9$ \\
\hline Aortic oxygen saturation (\%) & $77 \pm 7$ & $75 \pm 5$ \\
\hline Pulmonary artery pressure $(\mathrm{mm} \mathrm{Hg})$ & $15 \pm 7$ & $13 \pm 3$ \\
\hline Left atrial pressure $(\mathrm{mm} \mathrm{Hg})$ & $7 \pm 3$ & $7 \pm 3$ \\
\hline Ventricular end-diastolic pressure (mm Hg) & $8 \pm 2$ & $7 \pm 3$ \\
\hline Pulmonary blood flow $(\mathrm{Qp})\left(\mathrm{L} \cdot \mathrm{min}^{-1} \cdot \mathrm{m}^{-2}\right)$ & $4.5 \pm 2.3$ & $3.7 \pm 1.4$ \\
\hline Systemic blood flow (Qs) $\left(\mathrm{L} \cdot \mathrm{min}^{-1} \cdot \mathrm{m}^{-2}\right)$ & $3.4 \pm 1.1$ & $3.1 \pm 1.3$ \\
\hline $\mathrm{Qp} / \mathrm{Qs}$ & $1.5 \pm 1.1$ & $1.2 \pm 0.8$ \\
\hline \multicolumn{3}{|l|}{ Operative } \\
\hline Total support time (min) & $76 \pm 39(n=51)$ & $74 \pm 22(n=79)$ \\
\hline Circulatory arrest time (min) & $15 \pm 20(n=43)$ & $35 \pm 13(\mathrm{n}=79)$ \\
\hline \multicolumn{3}{|l|}{ Postoperative day 1} \\
\hline Cavopulmonary pressure $(\mathrm{mm} \mathrm{Hg})$ & $11 \pm 2$ & $10 \pm 3$ \\
\hline Left atrial pressure $(\mathrm{mm} \mathrm{Hg})$ & $7 \pm 3$ & $6 \pm 2$ \\
\hline Aortic oxygen saturation (\%) & $78 \pm 7$ & $78 \pm 6$ \\
\hline Duration ventilated $(\mathrm{h})^{*}$ & $6(3-77)$ & $7(3-312)$ \\
\hline Duration chest tube drainage (d)* & $1(1-19)$ & $1(1-27)$ \\
\hline Duration hospitalization $(\mathrm{d}) *$ & $5(3-70)$ & $5(2-28)$ \\
\hline
\end{tabular}

Total support time includes cardiopulmonary bypass time and circulatory arrest time. $B D G$, Bidirectional Glenn; $H F$, hemi-Fontan; $S V C$, superior vena cava; $C P A$ cavopulmonary anastomosis.

* Median and range data shown.

Fontan operation (29 patients, 36\%) than after the bidirectional Glenn shunt (5 patients, $9.8 \%$; relative risk [RR]: 3.7; confidence interval [CI]: 1.5-8.9) (Fig 2). However, by hospital discharge this difference was no longer apparent. At hospital discharge, sinus node dysfunction persisted in only 3 patients $(6 \%)$ with a bidirectional Glenn shunt and in $7(8 \%)$ after the hemiFontan operation. Of the 7 patients with sinus node dysfunction after the hemi-Fontan procedure, 1 patient had sinus bradycardia and the remaining 6 patients were in a predominant junctional rhythm. All 3 patients with persistent sinus node dysfunction after the bidirectional Glenn shunt had junctional rhythm. Ectopic atrial rhythm was present in 5 patients at hospital discharge after the cavopulmonary anastomosis (4 in the bidirectional Glenn group and 1 in the hemi-Fontan group). Temporary atrial pacing for junctional rhythm to augment cardiac output was used in 3 patients (bidirectional Glenn, $\mathrm{n}=1$; hemi-Fontan, $\mathrm{n}=2$ ).

Of the 10 patients who were discharged with sinus node dysfunction after undergoing intermediate cavopulmonary connection, 6 are awaiting Fontan surgery, and 4 have had completion of the Fontan repair. All 4 of these patients returned for the Fontan procedure in sinus rhythm without any evidence of bradycardia or junctional rhythm on their ambulatory monitors. Three additional patients (1 bidirectional
Glenn group; 2 hemi-Fontan group) who were discharged in sinus rhythm after undergoing the superior cavopulmonary anastomosis presented for the Fontan operation with evidence of sinus node dysfunction. Of these 3 patients, 2 had sinus bradycardia and 1 had junctional rhythm.

Sinus node dysfunction was observed in $8(26 \%)$ patients on the first postoperative day after the extracardiac conduit but remained in only $4(13 \%)$ by hospital discharge. In contrast, early sinus node dysfunction was observed in $6(13 \%)$ patients after the lateral tunnel Fontan procedure and persisted in 4 patients at hospital discharge (Fig 3). In 2 additional patients undergoing the lateral tunnel Fontan operation, sinus node dysfunction developed between the first postoperative day and hospital discharge. There was no significant difference in the incidence of sinus node dysfunction at discharge after the completion of either the bidirectional Glenn/extracardiac conduit $(13 \%)$ or the hemi-Fontan/lateral tunnel Fontan operation $(13 \%)$. Sinus node dysfunction at discharge after the Fontan operation manifested as junctional rhythm in 8 patients, junctional bradycardia in 1 patient, and sinus bradycardia in 1 patient. Ectopic atrial rhythm was present in 6 patients after the Fontan operation (3 bidirectional Glenn/extracardiac conduit; 3 hemi-Fontan/lateral tunnel). 
Table III. Fontan procedure: Preoperative and perioperative hemodynamics

\begin{tabular}{lcc}
\hline & $B D G / E C(n=30)$ & $H F / L T(n=46)$ \\
\hline Perioperative & & \\
Pre-Fontan cardiac catheterization & & $59 \pm 8$ \\
SVC saturation (\%) & $57 \pm 10$ & $83 \pm 5$ \\
Aortic oxygen saturation (\%) & $82 \pm 7$ & $11 \pm 4$ \\
Pulmonary artery pressure (mm Hg) & $10 \pm 3$ & $6 \pm 3$ \\
Left atrial pressure (mm Hg) & $6 \pm 2$ & $7 \pm 2$ \\
Ventricular end-diastolic pressure $\left(\mathrm{mm} \mathrm{Hg}^{-1}\right)$ & $7 \pm 2$ & $2.3 \pm 0.7$ \\
Pulmonary blood flow (Qp) $\left(\mathrm{L} \cdot \mathrm{min}^{-1} \cdot \mathrm{m}^{-2}\right)$ & $2.6 \pm 1.5$ & $3.5 \pm 1.1$ \\
Systemic blood flow (Qs) (L $\left.\cdot \mathrm{min}^{-1} \cdot \mathrm{m}^{-2}\right)$ & $3.5 \pm 0.9$ & $0.7 \pm 0.1$ \\
Qp/Qs & $0.7 \pm 0.3$ & $57 \pm 8$ \\
Operative & & $18 \pm 8$ \\
Total support time (min) & $103 \pm 33$ & $11 \pm 3$ \\
Circulatory arrest time (min) & $23 \pm 22$ & $8 \pm 3$ \\
Postoperative day 1 & & $87 \pm 6$ \\
Cavopulmonary pressure (mm Hg) & $12 \pm 4$ & $5(3-141)$ \\
Left atrial pressure (mm Hg) & $8 \pm 3$ & $1(1-14)$ \\
Aortic oxygen saturation (\%) & $87 \pm 9$ & 5 \\
Duration ventilated (h)* & $5(2-340)$ & $5(3-55)$ \\
Duration chest tube drainage (d)* & $1(1-24)$ & 6 \\
Chest tube duration $>$ 7 d (No.) & 6 & \\
Duration hospitalization (d)* & $6(3-56)$ & \\
\hline
\end{tabular}

Total support time includes cardiopulmonary bypass time and circulatory arrest time. $B D G$, Bidirectional Glenn; $H F$, hemi-Fontan; $E C$, extracardiac conduit Fontan; $L T$, lateral tunnel Fontan.

*Median and range data shown

Among the 130 patients, the overall incidence of early sinus node dysfunction was $13 \%$. Among those who had at least one operation that might jeopardize the sinus node artery, the incidence of sinus node dysfunction was $12.7 \%$ (10/79); among those having no operations thought to jeopardize the sinus node, the incidence of sinus node dysfunction was $13.7 \%$ (7/51). The relative risk for sinus node dysfunction in the "exposed" group was 0.92 (CI: $0.38-2.27 ; P=\mathrm{NS}$ ). No discernible difference was found in the incidence of sinus node dysfunction among patients having both a hemi-Fontan and lateral tunnel operation compared with those having one or neither sinus node-jeopardizing procedures (RR: 0.99 ; CI: $0.38-2.5 ; P=\mathrm{NS}$ ). There were no hemodynamic or perioperative predictors of sinus node dysfunction. Despite the longer bypass times in patients having a hemi-Fontan operation, no effect was observed on the development of sinus node dysfunction. No difference in the incidence of sinus node dysfunction was discernible among patients classified as having heterotaxy syndrome (RR: 1.1; CI: 0.39-2.9; $P=\mathrm{NS}$ ).

Epicardial pacemakers were placed in 3 patients in the study cohort. One patient had a malaligned atrioventricular canal, hypoplastic right ventricle, and small tricuspid valve. A biventricular repair was attempted in the neonatal period with closure of the ventricular septal defect. The operation was complicated by complete heart block necessitating placement of a ventricular demand pacemaker. On discontinuation of cardiopulmonary bypass, the patient was hypotensive and in low cardiac output with evidence of significant tricuspid stenosis. The ventricular septal defect patch was taken down and a pulmonary artery band was constructed. The child went on to have a hemi-Fontan and subsequent lateral tunnel Fontan operation. This patient had normal atrial rates on Holter monitors without sinus node dysfunction. The second patient had evidence of Mobitz I atrioventricular block and prolonged pauses after the Norwood operation, prompting placement of a single-chamber ventricular pacemaker. This patient also had normal atrial rates on ambulatory monitors. At the time of the hemi-Fontan operation, the pacemaker was upgraded to a dual-chamber (DDD) epicardial system. The third child had a dual-chamber pacemaker placed at the time of the lateral tunnel Fontan operation because of prolonged sinus pauses noted on a screening ambulatory monitor. This patient met the criteria for sinus node dysfunction.

Arrhythmias after both the superior cavopulmonary anastomosis and the Fontan procedure were rare, although slightly more common after the hemi-Fontan 


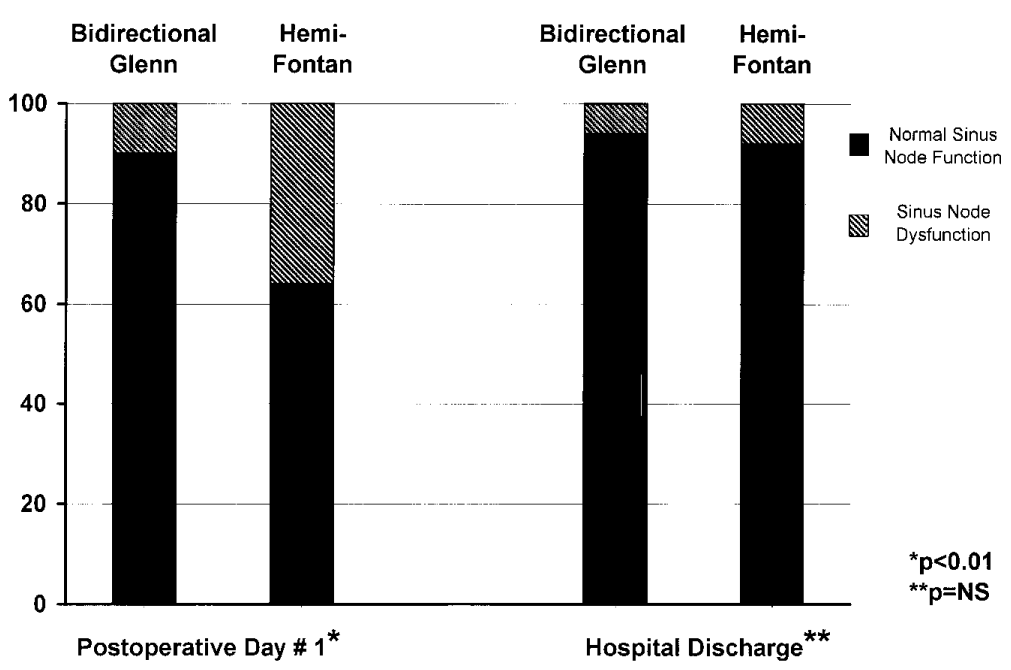

Fig 2. Sinus node function on postoperative day 1 and hospital discharge after the superior cavopulmonary anastomosis. All 130 patients (51 bidirectional Glenn and 79 hemi-Fontan procedures) were included. There was a significant difference $(P<.01)$ in the incidence of sinus node dysfunction on the first postoperative day after the bidirectional Glenn compared with the hemi-Fontan operation. By hospital discharge no discernible difference in sinus node dysfunction was observed. Black columns, Normal sinus node function; hatched columns, sinus node dysfunction. $N S$, Not significant.

procedure. One patient had nonsustained supraventricular tachycardia after the bidirectional Glenn shunt and did not require any treatment. Supraventricular tachycardia was noted in 3 patients after the hemi-Fontan operation and included orthodromic re-entrant tachycardia in 1 patient and ectopic atrial tachycardia in 2 patients. All 3 patients received antiarrhythmic medication (digoxin in 1; amiodarone in 2) for 6 months with no subsequent recurrences of tachycardia. Junctional ectopic tachycardia occurred after the hemi-Fontan procedure in 2 patients and after the Fontan operation in 3 patients. All 5 patients were successfully managed with surface cooling, amiodarone, and temporary atrial pacing. Two patients had nonsustained supraventricular tachycardia that did not necessitate any antiarrhythmic therapy. One patient returned within 30 days of the extracardiac conduit procedure with atrial flutter and rapid ventricular conduction. This patient was successfully cardioverted to sinus rhythm, treated with amiodarone, and has remained free of atrial arrhythmias over the past 2 years.

Hospital course. The median length of hospitalization after the Fontan operation was 5 days (3-56 days). Chest tubes were removed in $76 \%$ of patients by the third postoperative day after the Fontan operation. Only 8 patients had pleural drainage exceeding 1 week (Table III). No significant difference was observed between any of the surgical groups with respect to the duration of chest tube drainage, incidence of pleural effusions, or hospitalization. After an extracardiac Fontan procedure, 1 patient was readmitted with a pericardial effusion on postoperative day 12 and underwent a pericardiocentesis. Seven patients in the study cohort were readmitted within the first 30 days. Five patients were readmitted with effusions after an extracardiac Fontan operation. Pleural catheters were placed in 2 of these patients and the other 3 were successfully managed with diuretics. Two patients having the lateral tunnel Fontan operation were readmitted with pleural effusions and 1 underwent placement of a chest tube. The 1 patient who died after the Fontan operation (mortality 1.3\%) had innominate vein thrombosis and cyanosis after the bidirectional Glenn shunt, and the operation had to be converted to a stage I Norwood before the Fontan operation. No patients in this cohort underwent a Fontan takedown procedure or heart transplantation during the study period.

\section{Discussion}

This study revealed a similar incidence (8\%) of sinus node dysfunction at hospital discharge in patients undergoing either a bidirectional Glenn shunt or a hemi-Fontan operation. The incidence of sinus node dysfunction increased to $13 \%$ after either an extracar- 


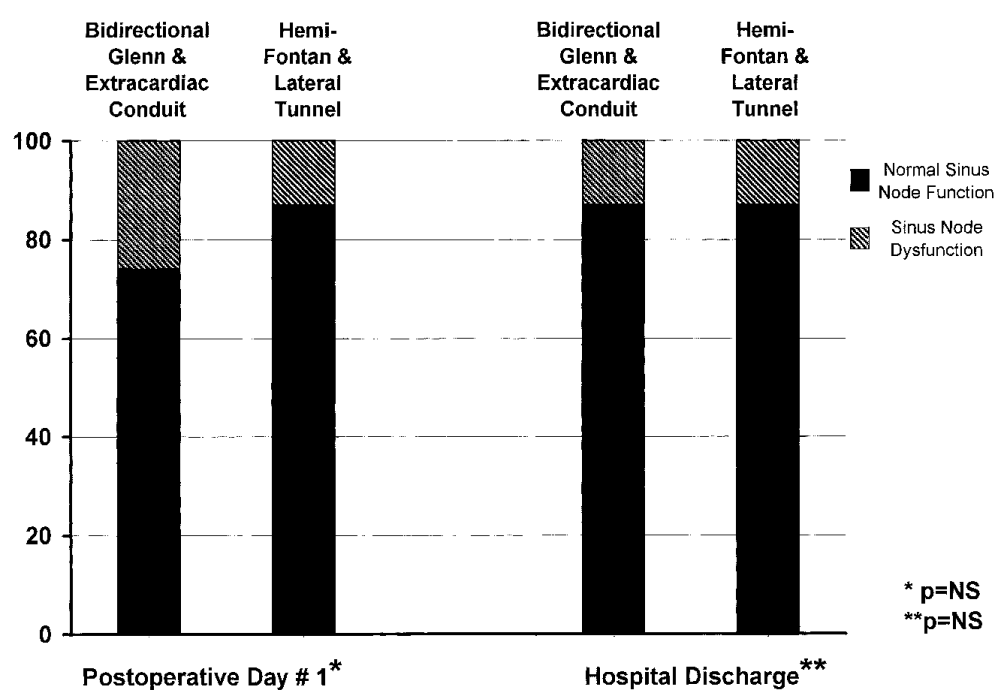

Fig 3. Sinus node function on postoperative day 1 and hospital discharge after the Fontan operation. Data are shown on 30 patients after the extracardiac Fontan and on 46 patients after the lateral tunnel Fontan operation. The two patients with an initial hemi-Fontan and subsequent extracardiac conduit were excluded. There was no discernible difference in the incidence of sinus node dysfunction on the first postoperative day or hospital discharge between the two staged techniques. Black columns, Normal sinus node function; hatched columns, sinus node dysfunction. $N S$, Not significant.

diac or lateral tunnel Fontan procedure with no discernible difference between the two groups.

Sinus node dysfunction may be particularly troublesome in children with Fontan physiology. Cardiac output may be limited in patients with a relatively slow heart rate and fixed stroke volume. In addition, junctional rhythm may reduce cardiac output by not providing a synchronous atrial contraction before ventricular systole. Finally, sinus node dysfunction may predispose patients to the development of atrial flutter, a cause of significant morbidity and mortality in this population. ${ }^{16}$

Multiple definitions of sinus node dysfunction have been proposed. Although there is little controversy that a patient with junctional bradycardia and sinus pauses exceeding 3 seconds has sinus node dysfunction, it may be less clear in the patient with a heart rate just slightly below the age-adjusted mean. In addition, previous retrospective studies evaluating sinus node dysfunction in this patient population have used ECGs and Holter monitors only if clinically indicated. ${ }^{8,16}$ The noninvasive assessment of sinus node function is generally considered to be as reliable as more invasive diagnostic tools. ${ }^{17,18}$ Furthermore, because the incidence of sinus node dysfunction increases with time from surgery, any comparison of surgical modifications from different eras must be adjusted for duration of follow-up. In an attempt to address these multiple variables, this prospective study compared the early incidence of sinus node dysfunction between the hemi-Fontan/lateral tunnel approach and the bidirectional Glenn/extracardiac conduit Fontan with the use of routine ECGs and ambulatory Holter monitors at each surgical step. Discharge ambulatory Holter monitors were available in $93 \%$ of the study cohort. We found no discernible difference among those patients who had at least one operation that might jeopardize the sinus node (either hemi-Fontan and/or lateral tunnel Fontan) compared with those having no operations thought to jeopardize the sinus node (specifically, the bidirectional Glenn and/or extracardiac conduit).

Although both the hemi-Fontan and bidirectional Glenn procedures remove the adverse effects of prolonged ventricular volume load and allow time for resolution of the ventricular hypertrophy before the Fontan operation, the two surgical techniques are quite different. As it is performed at our institution, the bidirectional Glenn shunt consists of transection of the SVC 5 to $10 \mathrm{~mm}$ above the right atrial junction, anastomosis to the ipsilateral pulmonary artery, and oversewing of the cardiac end of the SVC. This is distinctly different from the hemi-Fontan operation, in which an incision is made in the superior part of the atrium, near the sinus node, and extended onto the 
medial aspect of the SVC. Although the hemi-Fontan operation facilitates completion of the lateral tunnel, because only the "cavoatrial dam" needs to be removed at the time of the Fontan operation, these cavoatrial incisions expose the sinus node and sinus node artery to injury. ${ }^{4,19}$ Concern has been raised that the superior cavopulmonary connection in combination with the lateral tunnel Fontan further increases the risk of sinus node dysfunction because of repeated dissection near the sinoatrial node and manipulation of the atrium. ${ }^{8}$

$\mathrm{We}^{9}$ previously reported a $23 \%$ incidence of sinus node dysfunction early after the staged lateral tunnel Fontan that further increased in occurrence from the time of the Fontan operation. In an attempt to avoid injury to the sinoatrial node arterial supply, Douglas and colleagues ${ }^{20}$ further modified the hemi-Fontan procedure by "offsetting" the cavoatrial incision. In these patients, an incision was made in the base of the right atrial appendage toward the SVC, but avoided crossing the cavoatrial junction. Although the prevalence of sinus rhythm on the immediate postoperative ECG improved with this modified technique, no difference was observed between the standard and modified hemi-Fontan procedures by hospital discharge. This is consistent with our observation of a greater incidence of sinus node dysfunction on the first postoperative day after the hemi-Fontan operation compared with the bidirectional Glenn shunt, but without a discernible difference by hospital discharge.

There is speculation that avoiding the sinus node region altogether reduces the likelihood of sinus node dysfunction developing. The bidirectional Glenn procedure and subsequent extracardiac conduit, so-called "atrial no-touch surgery," avoid exposing the right atrium to elevated systemic venous pressure, surgery near the sinus node, and limit the extensive atrial suture lines and scarring commonly seen with the lateral tunnel Fontan operation. Petrossian and colleagues ${ }^{10}$ retrospectively reported on 51 patients who underwent an extracardiac Fontan operation. Approximately $80 \%$ of these patients had a previous bidirectional Glenn anastomosis and none had a hemiFontan procedure. Transient sinus node dysfunction developed in $8 \%$ of these patients in the early postoperative period after the extracardiac conduit. In a slightly smaller retrospective study evaluating the extracardiac conduit Fontan procedure in 16 patients, Shirai and coworkers ${ }^{21}$ found that $44 \%$ had sinus node dysfunction on ambulatory monitors. Both studies were retrospective and did not have a contemporaneous group of patients undergoing a hemi-Fontan/lateral tunnel Fontan operation for comparison.
In addition to the similar prevalence of early sinus node dysfunction, the surgical groups in this study were quite similar in their operative and postoperative courses. We observed no significant difference in the length of hospitalization, duration of effusions, or perioperative hemodynamics with either staged Fontan procedure. The mortality after the superior cavopulmonary anastomosis was $2.3 \%$ and after the Fontan operation, $1.3 \%$. In addition, no early difference was found in the incidence of postoperative tachyarrhythmias between the surgical groups. In fact, the 1 patient in whom atrial flutter developed within the first 30 days of the Fontan operation had undergone an initial bidirectional Glenn shunt and subsequent extracardiac conduit.

Study limitations. Factors such as details of cannulation, cardiopulmonary bypass, myocardial protection, atrial suture lines, manipulation of the cavae, or other unidentified etiologies may explain the similar early incidence of sinus node dysfunction between the two staged Fontan techniques, but these were not addressed with this study design. Too few patients underwent a nonstaged Fontan operation during this time period to make a comparative analysis meaningful.

\section{Conclusions}

In this prospective cohort study, avoidance of surgery near the sinus node had no discernible effect on the development of early sinus node dysfunction. Thus, concerns about early sinus node dysfunction should not override patient anatomy or surgeon preference as determinants of which superior cavopulmonary anastomosis and Fontan procedure to perform. A longer duration of follow-up with exercise testing to assess chronotropic competence is clearly indicated in these patients, as one surgical staging approach may prove more beneficial and reduce the long-term incidence of sinus node dysfunction and atrial arrhythmias.

\section{REFERENCES}

1. Bridges ND, Jonas RA, Mayer JE, Flanagan MF, Keane JF, Castaneda AR. Bidirectional cavopulmonary anastomosis as interim palliation for high-risk Fontan candidates. Circulation 1990;82(Suppl):IV-170-6.

2. Lamberti JJ, Spicer RL, Waldman JD, et al. The bidirectional cavopulmonary shunt. J Thorac Cardiovasc Surg 1990;100:2230.

3. Bradley SM, Mosca RS, Hennein HA, Crowley DC, Kulik TJ, Bove EL. Bidirectional superior cavopulmonary connection in young infants. Circulation 1996;94(Suppl):II-5-11.

4. Jacobs ML, Norwood WI Jr. Fontan operation: influence of modifications on morbidity and mortality. Ann Thorac Surg 1994;58:945-52.

5. Rychik J, Jacobs ML, Norwood WI Jr. Acute changes in left ven- 
tricular geometry after volume reduction operation. Ann Thorac Surg 1995;60:1267-74.

6. Forbes JM, Cook N, Serraf A, Burke RP, Mayer JE, Jonas RA. An institutional experience with second- and third-stage palliative procedures for hypoplastic left heart syndrome: the impact of the bidirectional cavopulmonary shunt. J Am Coll Cardiol 1997;29:665-70.

7. Koutlas TC, Gaynor JW, Nicolson SC, Steven JM, Wernovsky G, Spray TL. Modified ultrafiltration reduces postoperative morbidity after cavopulmonary connection. Ann Thorac Surg 1997;64:37-42.

8. Manning PB, Mayer JE Jr, Wernovsky G, Fishberger SB, Walsh EP. Staged operation to Fontan increases the incidence of sinoatrial node dysfunction. J Thorac Cardiovasc Surg 1996;111:833-40.

9. Cohen MI, Wernovsky G, Vetter VL, et al. Sinus node function after a systematically staged Fontan procedure. Circulation 1998;98(Suppl):II-352-9.

10. Petrossian E, Reddy VM, McElhinney DB, et al. Early results of the extracardiac conduit Fontan operation. J Thorac Cardiovasc Surg 1999;117:688-96.

11. Marcelletti CF, Iorio FS, Abella RF. Late results of extracardiac Fontan repair. Pediatr Card Surg Annu Semin Thorac Cardiovasc Surg 1999;2:131-41.

12. Spray TL. Fenestrated Fontan for hypoplastic left heart syndrome. Operative Techniques Card Thorac Surg 1997;2:239-52.

13. Romano M, Clarizia M, Onofrio E, et al. Heart rate, PR, and QT intervals in normal children: a 24-hour Holter monitoring study. Clin Cardiol 1988;11:839-42.

14. Garson A Jr. Electrocardiography. In: Garson A Jr, Bricker TJ, McNamara DG, editors. The science and practice of pediatric cardiology. Malvern [PA]: Lea \& Febiger; 1990. p. 714.

15. Gentles TL, Mayer JE Jr, Gauvreau K, et al. Fontan operation in five hundred consecutive patients: factors influencing early and late outcome. J Thorac Cardiovasc Surg 1997;111:376-91

16. Fishberger SB, Wernovsky G, Gentles TL, et al. Factors that influence the development of atrial flutter after the Fontan operation. J Thorac Cardiovasc Surg 1997;113:80-6.

17. Reiffel JA, Bigger JT Jr, Cramer M, et al. Ability of Holter electrocardiographic recording and atrial stimulation to detect sinus nodal dysfunction in symptomatic and asymptomatic patients with sinus bradycardia. Am J Cardiol 1977;40:189-93.

18. Thormann J, Schlepper M, Kramer W. Diurnal changes and reproducibility of corrected sinus node recovery time. Cathet Cardiovasc Diagn 1983;9:439-43.

19. Norwood WI Jr, Jacobs ML, Murphy JD. Fontan procedure for hypoplastic left heart syndrome. Ann Thorac Surg 1992;54:102530.

20. Douglas WI, Goldberg CS, Mosca RS, Law IH, Bove EL. HemiFontan procedure for hypoplastic left heart syndrome: outcome and suitability for Fontan. Ann Thorac Surg 1999;68:1361-8.

21. Shirai LK, Rosenthal DN, Reitz BA, Robbins RC, Dubin AM. Arrhythmias and thromboembolic complications after the extracardiac Fontan operation. J Thorac Cardiovasc Surg 1998;115:499-505.

\section{Discussion}

Dr Peter Manning (Cincinnati, Ohio). Studying postoperative complications after Fontan reconstruction has been a notoriously difficult endeavor. Most of the significant complications are noted to increase in frequency with prolonged follow-up or may not be noted at all until the late follow-up period. By the time we evaluate how they relate to our surgical techniques, our methods have typically changed. We are faced with the challenge of studying a constantly moving target.

This study has a number of exceptional strengths. The authors have clearly defined what they are calling sinus node dysfunction and have collected their data prospectively. The patients fell into two fairly discrete management pathways, although I am unsure how they were chosen for these pathways. Were they randomized or was this a change in practice over time?

The authors also were able to enroll a fairly large number of patients over a fairly short period. As the incidence of problems has been widely documented to increase with the duration of follow-up, the short-term nature of the study, as the authors mentioned, is certainly a limitation; however, other studies have shown that early sinus node dysfunction is in fact a predictor of late rhythm problems.

Although this study does represent the two currently most commonly followed methods for staged Fontan reconstruction, other techniques are also frequently used. In the study that the authors cited from Children's Hospital in Boston, in which I was involved, most patients underwent an initial bidirectional Glenn shunt followed by a lateral tunnel procedure. This is a different scheme from that which their group studied, and I believe it exposes the sinus node to a greater degree of injury at the time of Fontan completion than either strategy that the authors used, due to the need to create an anastomosis between the roof of the atrium and the pulmonary artery.

The authors seem to conclude that techniques avoiding trauma to the sinus node area do not influence the development of sinus node dysfunction. However, I conclude that they have achieved fairly exemplary, yet similar, results using two techniques, both of which try to limit the dissection and injury to the sinus node, albeit one more other than the other, at the intermediate stage. The incidence of early sinus node dysfunction with either of these methods was half of what we reported using the sequence of bidirectional Glenn shunting followed by a lateral tunnel reconstruction.

I have three questions for the authors.

I noted that the rate of sinus node dysfunction in the group undergoing hemi-Fontan after lateral tunnel completion was quite a bit lower than that of the similarly managed group that you had previously reported. That group of patients was similarly managed, but from a period 5 years preceding the current study. How do you explain that apparent difference in the incidence? Was there a modification in the surgical technique?

Do you believe that despite the best surgical efforts we must accept a certain incidence of sinus node dysfunction in this patient population? Furthermore, do you believe that factors other than surgical trauma influence the development of this complication?

Third, have you been able to study the physiologic impact of sinus node dysfunction in the palliated single ventricle physiology, or have you any recommendations regarding the indications for considering permanent pacing in these children? 
Dr Cohen. Dr Manning, thank you for your comments.

To address the first comment, the patients were not randomized. We have 4 surgeons at our institution and the decision of which cavopulmonary connection to perform was essentially at their discretion. However, patients with heterotaxy syndrome with anomalies of the IVC and SVC were more likely to have a bidirectional Glenn and extracardiac conduit Fontan operation.

I will now address the remaining questions at the end of your discussion. The difference in sinus node dysfunction between this current study and the report that we published a few years ago is not due to a change in the surgical technique, but in the way in which we have progressed to evaluating sinus node function. The initial study was a retrospective study in which very often the perioperative rhythm was only assessed on the first postoperative day. Patients did not have discharge ambulatory Holter monitors. I believe a fair number of children are in junctional rhythm on their first postoperative day, but subsequently return to sinus rhythm when they are evaluated 5 days later with a Holter monitor.

In answer to the second question, I believe that the etiology of sinus node dysfunction is more than simple injury to the sinus node artery at the time of the cavoatrial incision. If it were something that simple, then all of the patients in our hemi-Fontan group, at least the way it is performed at our institution, should have sinus node dysfunction. Clearly, neither this current study nor our previous article supports that observation. The cause of sinus node dysfunction in this patient population may relate to injury to the sinus node artery, sinus node nerve, disruption in autonomic input, suture lines, scarring, or it may be that sinus node dysfunction is intrinsic to patients with single ventricles. Further work regarding the etiology of sinus node dysfunction is needed.

With regard to the last question, there has been discussion at our institution about placing atrial pacemakers at the time of the Fontan operation to prevent any bradycardia. From an electrophysiology standpoint, these patients are at risk for the development of atrial flutter. The presence of bradycardia in the face of conduction barriers caused by suture lines and scarring may allow premature atrial beats to initiate intra-atrial re-entrant tachycardia. There is some speculation that if bradycardia is prevented, the late incidence of atrial flutter may be reduced.

\section{THE THORACIC SURGERY FOUNDATION FOR RESEARCH AND EDUCATION}

\section{Datascope Makes Major Gift}

The Thoracic Surgery Foundation is most pleased to announce that Datascope Corporation recently pledged $\$ 1$ million toward the $\$ 3.75$ million in matching funds needed for The Foundation to secure a $\$ 5$ million grant from the National Heart, Lung, and Blood Institute. These funds will be used to support the jointly sponsored TSFRE and NHLBI Mentored Clinical Scientist Development Award Program. The ten-year program is designed to develop outstanding cardiothoracic surgeon-scientists.

Datascope's gift to The Thoracic Surgery Foundation is a very important step forward in the TSFRE's efforts to secure the NHLBI funds. In 1999, the von Liebig Foundation also agreed to support The Foundation in this initiative if The Foundation could find other partners, such as Datascope, to commit to the matching funds.

The Foundation in extremely grateful to Datascope and to Mr Lawrence Saper, President and CEO of Datascope, for this very generous support. 